\title{
1 Die Differenz macht den Unterschied: Differenzhermeneutische Ansätze in der Religionstheologie
}

Die konfessionsübergreifend in der Theologie geführte Debatte um eine Theologie der Religionen hat in den letzten Jahrzehnten mehrere Programme hervorgebracht, die sich in Ansatz, Methode und Zielbestimmung markant voneinander unterscheiden. Sieht man vom „Projekt Weltethos“ ab, das sich von der Religionstheologie und -hermeneutik distanziert und sich darauf beschränken will, nach Gemeinsamkeiten im Ethos der Religionstraditionen zu suchen, so sind dies vor allem die „Pluralistische Religionstheologie“, ${ }^{1}$ die „Komparative Theologie“2 und die unter dem von Christian Danz vorgeschlagenen Begriff des „neuen Inklusivismus“3 ${ }^{3}$ zusammengefassten religionshermeneutisch ansetzenden Entwürfe. Das jüngste Mitglied dieser Familie ist die „Differenzhermeneutik“.

Doch schon der erste Blick auf die sich mit diesem Etikett bezeichnenden Entwürfe zeigt eine große Heterogenität. Ich stelle im Folgenden sechs Ansätze vor, die den Differenzbegriff auf zum Teil unterschiedliche Weise, in differierenden Begründungszusammenhängen und mit unterschiedlicher Absicht gebrauchen.

\section{Differenzhermeneutik als Verstehen des Fremden}

Wenn Theo Sundermeier im Rahmen seiner „Hermeneutik des Fremden“ ${ }^{\star 4}$ den Begriff der „Differenzhermeneutik“ gebraucht, so verbindet sich damit die

1 Exemplarisch nenne ich nur: Perry Schmidt-Leukel, Gott ohne Grenzen. Eine christliche und pluralistische Theologie der Religionen (Gütersloh: Gütersloher Verlagshaus, 2005).

2 Ich sehe hier von der (unter den Vertretern dieser Richtung umstrittenen) Frage ab, ob es sich dabei überhaupt um einen Ansatz der Religionstheologie und nicht nur um eine religionsvergleichende Methode handelt. Gerade diejenigen Repräsentanten, die sich am stärksten von der Religionstheologie distanzier(t)en, haben in jüngster Vergangenheit Schriften publiziert, die eindeutig religionstheologischer Natur sind: Klaus von Stosch, Komparative Theologie als Wegweiser in der Welt der Religionen (Paderborn et al.: Schöningh, 2012); Francis X. Clooney, Comparative Theology. Deep Learning across Religious Borders (Chichester: Wiley-Blackwell, 2010).

3 Vgl. exemplarisch Reinhold Bernhardt, Ende des Dialogs? Die Begegnung der Religionen und ihre theologische Reflexion (Zürich: Theologischer Verlag, 2006).

4 Theo Sundermeier und Werner Ustorf (Hg.), Begegnung mit dem Anderen. Plädoyers für eine interkulturelle Hermeneutik (Gütersloh: Gütersloher Verlagshaus, 1991); Theo Sundermeier 
Forderung, die Selbstreferenzialität in der Wahrnehmung anderer Religionen möglichst aufzubrechen und deren genuine Andersheit zu würdigen. Es geht ihm um eine „Differenzhermeneutik, die das Differente verstehen lehrt, ohne es zu vereinnahmen, die praktische Hilfe bietet, die Nähe des Zusammenlebens einzuüben, und zugleich die richtige Distanz bewahrt, die die Identität des Fremden respektiert und die uns allen gemeinsame Menschenwürde achtet“. ${ }^{5}$

Das zu verhandelnde hermeneutische Problem betrifft im Kern die Frage, ob und unter welchen Bedingungen ein wirkliches Verstehen des Anderen als eines Anderen möglich ist, wo doch Verstehen immer auch mit der Einordnung in das eigene Vorverständnis einher geht, das dabei dann freilich einer Transformation unterzogen wird. Erklärtes Ziel der so verstandenen Differenzhermeneutik ist es, Vereinnahmungen des Anderen und Fremden durch das Aufsuchen des Eigenen in ihm oder durch die Projektion eigener Stilisierungen des Anderen zu vermeiden und sich ihm in seiner widerständigen Fremdheit auszusetzen. Aneignungshermeneutiken sind oft mit der Ausgrenzung derjenigen Anteile am Fremden verbunden, die nicht angeeignet werden können. Gerade solche Anteile aber versucht die Differenzhermeneutik in ihre Verstehensbemühungen mit einzubeziehen. Sundermeier stellt diesen Ansatz der gesamten - auf das Subjekt bzw. Selbst bezogenen - abendländischen Hermeneutiktradition gegenüber.

Der „Sitz im Leben“ dieses Ansatzes ist die Situation kultureller und religiöser Vielfalt. So schreibt Theo Sundermeier:

Die Notwendigkeit einer Hermeneutik interkulturellen Verstehens ist nicht geboren aus dem Streben nach mehr Wissen, nach Exotik, aus dem Bewusstsein heraus, dass wir mit unserem Wissen allein die gegenwärtigen Weltprobleme nicht bewältigen können. Nicht Defiziterfahrungen begründen also eine Hermeneutik, sondern die Erfahrung gemeinsamen Lebens. [...] Damit wird das Verstehen zur fundamentalen, allem Handeln vorangehenden Aufgabe. ${ }^{6}$

Dieser Ansatz verfolgt also eine praktische Absicht: Er soll „[d]ie Identität der sich Begegnenden“ gewährleisten, „ihre unaufkündbare Zusammengehörigkeit und ein Aufeinanderangewiesensein, das zur Anerkennung führt“. „Das kommt der Verwirklichung eines Paradoxes gleich: Bei sich selbst und gleichzeitig beim Fremden sein, Fremdheit akzeptieren, die dennoch Vertrautheit nicht

(Hg.), Den Fremden wahrnehmen. Bausteine für eine Xenologie (Gütersloh: Gütersloher Verlagshaus, 1992); ders., Den Fremden verstehen. Eine praktische Hermeneutik (Göttingen: Vandenhoeck \& Ruprecht, 1996).

5 Sundermeier, Den Fremden verstehen (wie Anm. 4), 13.

6 Theo Sundermeier, „Erwägungen zu einer Hermeneutik interkulturellen Verstehens“, in ders. und Ustorf (Hg.), Begegnung mit dem Anderen (wie Anm. 4), 13-28, hier 20. 
unmöglich macht, Distanz halten, die Nähe ist und ein Mitsein mit dem anderen einschließt.“7

Den theoretischen Bezugsrahmen dieses Ansatzes bildet die interkulturelle Hermeneutik - sowohl innerhalb der Theologie ${ }^{8}$ als auch außerhalb: in der interkulturellen Philosophie und in der Kulturanthropologie. ${ }^{9}$ Für letztere hat Martin Fuchs die Bedeutung des Differenzbegriffs folgendermaßen auf den Punkt gebracht: „Differenz, wie auch immer jeweils verstanden (als substantieller Unterschied oder als Nichtidentität und ständiges Werden, als unbedingt, kontingent oder mediatisiert), bildet [...] die Grundvokabel der Kulturanthropologie.“10

Sundermeiers „Xenologie“ greift auf ein Verständnis von Identitätsbildung zurück, das von einer wechselseitigen Konstitution von Identität und Alterität ausgeht. Das Eigene (Selbst) bildet sich am Anderen und bleibt so auf dieses angewiesen. Unterschiedenheit und Zugehörigkeit können und müssen auf diese Weise zusammen gedacht werden. Es ist gerade die Differenz, die verbindet. Diese Verbindung bei bleibender Unterschiedenheit besteht in fortwährenden Kommunikationsprozessen. Sundermeier spricht mit Blick auf diesen Austausch analogisch von „Osmose“ und bezeichnet das dabei immer wieder neu anzustrebende Ziel als „Konvivenz“. Es handelt sich bei dieser Leitvorstellung um die Sozialform einer Gemeinschaft, deren Mitglieder sich gegenseitig helfen, miteinander lernen und feiern.

Hinsichtlich dieser Zielbestimmung kann man allerdings kritisch fragen, ob Sundermeiers Differenzhermeneutik nicht doch auf eine Aufhebung der Differenzen hinausläuft. So sehr es ihr um die Vermeidung einseitiger Vereinnahmungen des Anderen und Fremden geht, so sehr schweben ihr doch gemeinschaftliche Lebensformen vor, in denen aus Fremden Freunde werden. Dass Alterität Gemeinschaftsbildungen auch verhindern oder zerstören kann, tritt in dieser Sichtweise zurück. ${ }^{11}$

7 Sundermeier, Den Fremden verstehen (wie Anm. 4), 132.

8 Vgl. dazu Henning Wrogemann, Interkulturelle Theologie und Hermeneutik. Grundfragen, aktuelle Beispiele, theoretische Perspektiven (Gütersloh: Gütersloher Verlagshaus, 2012), 43-159; Volker Küster, Einführung in die Interkulturelle Theologie (Göttingen: Vandenhoeck \& Ruprecht, 2011), bes. 109-129; Klaus Hock, Einführung in die Interkulturelle Theologie (Darmstadt: Wissenschaftliche Buchgesellschaft, 2011).

9 Einen wichtigen Impuls hat Bernhard Waldenfels in seinem Buch Der Stachel des Fremden (Frankfurt am Main: Suhrkamp, 1990) gegeben.

10 Martin Fuchs, „Der Verlust der Totalität. Die Anthropologie der Kultur“, in Kulturwissenschaft. Felder einer prozeßorientierten wissenschaftlichen Praxis, hrsg. von Heide Appelsmeyer und Elfriede Billmann-Mahecha (Weilerswist: Velbrück Wissenschaft, 2001), 18-53, hier 21.

11 Vgl. dazu auch Andreas Grünschloß, Der eigene und der fremde Glaube. Studien zur interreligiösen Fremdwahrnehmung in Islam, Hinduismus, Buddhismus und Christentum (Tübingen: 
Ein zweiter Einwand betrifft die Frage, ob das Ideal, den Anderen bzw. das Andere in seiner Andersheit wahrzunehmen, wirklich erreicht werden kann. Dazu müsste das wahrnehmende Subjekt seinen ihm eigenen Standpunkt, sein Vorverständnis und seine Perspektive verlassen und sich ganz dem Anderen anverwandeln. Es gibt durchaus die Möglichkeit, sich emphatisch in den Anderen hineinzudenken und gewissermaßen mit seinen Augen $\mathrm{zu}$ sehen. Doch bleibt auch diese Kunst der Perspektivenübernahme immer an die eigene Prägung gebunden. Insofern kann das von Sundermeier anvisierte Ziel bestenfalls asymptotisch erreicht werden - womit nicht bestritten werden soll, dass es sich um ein erstrebenswertes Ziel handelt. Doch gilt: Auch eine xenologische Hermeneutik hat ihre Grenze am Anderen. Auch sie kommt ohne Vereinnahmungen nicht aus. Die Selbstreferenzialität der Wahrnehmung ist letztlich nicht aufhebbar.

Ein dritter Einwand bezieht sich auf das Verständnis des „Anderen“ und „Fremden“ bei Sundermeier. Zum einen werden die Begriffe weitgehend gleichbedeutend gebraucht. Hier wäre eine stärkere Unterscheidung geboten. Jeder mir Fremde ist auch ein Anderer, aber nicht jeder Andere auch ein mir Fremder. Zudem wäre zu präzisieren, ob es sich bei „Andersheit“ und „Fremdheit“ um abstrakte Kategorien der Erfahrung handelt („,ich erfahre etwas als fremd“) oder ob diese Begriffe eine „objektive“, also etwa ethnische, soziokulturelle oder religiöse Andersheit des Anderen bezeichnen. Offensichtlich fließt beides bei Sundermeier ineinander.

\section{Differenzhermeneutik als Wahrnehmung von Verschiedenheit}

Ulrich H. J. Körtner überträgt das Differenzparadigma von der ökumenischen Theologie auf die Religionstheologie. Insbesondere in seinem Buch Wohin steuert die Ökumene?, ${ }^{12}$ in dem er sich für die Ausbildung einer ökumenischen Hermeneutik aussprach, hatte er sich gegen „Konsens“ und „Konvergenz“ als Zielbestimmungen des ökumenischen Prozesses gewandt und stattdessen die

Mohr Siebeck, 1999), 308-309; Christian Danz, Einführung in die Theologie der Religionen (Wien: LIT-Verlag, 2005), 223-228.

12 Ulrich H. J. Körtner, Wohin steuert die Ökumene? Vom Konsens- zum Differenzmodell (Göttingen: Vandenhoeck \& Ruprecht, 2005); ders., Einführung in die theologische Hermeneutik (Darmstadt: Wissenschaftliche Buchgesellschaft, 2006), 164-171. 
Anerkennung bleibender Differenzen zwischen den christlichen Konfessionen gefordert. Damit knüpfte er an das von Harding Meyer propagierte Leitwort von der „versöhnten Verschiedenheit“ an, ${ }^{13}$ führt es aber weiter. Auch das Konzept des „differenzierten Konsenses“"14 ${ }^{14}$ war seiner Kritik ausgesetzt, weil aus seiner Sicht auch hier unklar blieb, welchen Stellenwert die verbleibenden Differenzen hätten, wenn sie (nach lehramtlicher römisch-katholischer Interpretation) nicht mehr zwangsläufig zu gegenseitigen Lehrverurteilungen führten, aber dennoch kirchentrennende Bedeutung behielten. Körtner sprach sich gegen das Modell einer uniformen Einheit und für eine in sich differente und komplexe Gemeinschaft der Kirchen mit ihren je eigenen Prägungen aus. ${ }^{15}$ In die gleiche Richtung tendierte auch Wolfgang Hubers „Ökumene der Profile“. ${ }^{16}$

Inähnlicher Weise führt Körtner das Differenzparadigma in die Religionstheologie ein: „Ausgangspunkt jeder Theologie der Religionen ist die Wahrnehmung von Verschiedenheit. “17 Doch weist er sogleich darauf hin, dass Differenzen nicht einfach objektiv vorliegen, sondern immer das Produkt einer Konstruktion des jeweiligen (individuellen oder kollektiven) Betrachters sind. Sie entstehen, wo und indem Unterscheidungen vorgenommen werden, sind also immer relational zu verstehen. Angehörige von Religionsformen bestimmen ihr Selbstverständnis durch Unterscheidungen von anderen. Solche differentiellen Selbstdefinitionen können, wie Körtner anhand von Galater 5,6 zeigt, auch die Überwindung vormaliger Unterscheidungen beinhalten. ${ }^{18}$

Das Ziel einer Theologie der Religionen sieht Körtner in der Entwicklung einer differenztheoretischen Hermeneutik, ,welche aus der Perspektive einer konkreten Religion die hermeneutischen Bedingungen des interreligiösen Gesprächs zu klären versucht“ ${ }^{19}$ Dass er mit dieser Zielbestimmung die Theologie der Re-

13 Harding Meyer, Versöhnte Verschiedenheit. Aufsätze zur ökumenischen Theologie, Bd. 2: Der katholisch/lutherische Dialog (Frankfurt am Main: Lembeck, 2000).

14 Bilaterale Arbeitsgruppe der Deutschen Bischofskonferenz und der Kirchenleitung der Vereinigten Evangelisch-Lutherischen Kirche Deutschlands, Communio sanctorum. Die Kirche als Gemeinschaft der Heiligen (Paderborn, Frankfurt am Main, 2000), 12.

15 Körtner, Wohin steuert die Ökumene? (wie Anm. 12), 30.

16 Wolfgang Huber, Im Geist der Freiheit. Für eine Ökumene der Profile (Freiburg im Breisgau: Herder, 2007).

17 Ulrich H. J. Körtner, „Synkretismus und Differenzwahrnehmung als Problem einer Theologie der Religionen, “in Theologie der Religionen. Positionen und Perspektiven evangelischer Theologie, hrsg. von Christian Danz und Ulrich H. J. Körtner (Neukirchen-Vluyn: Neukirchener Theologie, 2005), 57-76, hier 64.

18 Ebd., 65.

19 Ebd., 67. 
ligionen aber nicht auf eine Hermeneutik des interreligiösen Dialogs reduzieren will, zeigt sich daran, dass er offenbarungstheologische Überlegungen ins Feld führt. Mit der (pneumatologisch begründeten) Betonung der Universalität der Offenbarung bewegt er sich auf eine fundamentaltheologische Grundlegung der Religionstheologie zu. Die Uroffenbarung in Christus wird demnach in speziellen Offenbarungen in der Kraft des Geistes Gottes auch für Nichtchristen zum rechtfertigenden Glauben. Körtner verweist in diesem Zusammenhang auf die paulinische Interpretation des Glaubens Abrahams in Römer $4 .^{20}$ Wenn Gottes Liebe allen Menschen gelte, so könne ihre Ereignung nicht auf den christlichen Traditionszusammenhang beschränkt sein.

Zur Bearbeitung interreligiöser Differenzerfahrungen zieht Körtner zudem die Unterscheidung Luthers von gepredigtem und verborgenem Gott heran. Es müsse demnach von einem auf die gesamte Schöpfung bezogenen verborgenen Handeln Gottes ausgegangen werden. Daraus ergebe sich die Annahme von außerchristlichen Offenbarungen, die Christen als eigene Glaubenserfahrungen nicht zugänglich seien.

Diese - aus der Mitte der christlichen Theologie entnommenen - Deutungsmuster ermöglichen es Körtner, interreligiöse Differenzen stehen zu lassen, so dass sie weder zu Trennungen führen noch auf einer höheren Ebene harmonisiert oder gar synthetisiert werden müssen. Wichtig ist dabei seine konstruktivistische - und somit geschichtlich-dynamische und kontextrelative - Sicht der Differenzen. Diese bestehen nicht einfach, sondern ergeben sich in der Ausbildung und Weiterentwicklung von religiösen Selbstverständnissen. Sie können sich in den Traditionsströmen verfestigen und zu kollektiven Identitätsmarkern werden. Doch auch solche Verfestigungen sind dem Interpretationsstrom der Geschichte einer Religion ausgesetzt. Aus schneidenden Gegensätzen können komplementäre Polaritäten werden, deren Pole sich gegenseitig befruchten. Auch die Verfestigungen sind im Fluss.

Es gibt erhebliche Unterschiede zwischen den Differenzen, je nachdem, wie diese kodiert sind: ob als kreative Spannungen oder als trennende Frontstellungen. Doch auch solche Kodierungen lassen sich immer wieder verflüssigen. Es ist die Aufgabe der interreligiösen Hermeneutik, die Prozesse der Identitätsbestimmung durch Differenzmarkierung aufzuarbeiten und diese dabei zu entessenzialisieren. Wo ihr dies gelingt, erfüllt sie die ihr von Sundermeier zugedachte - praktische - Aufgabe, zur interreligiösen Verständigung beizutragen.

20 Ebd., 70-71. 


\section{Differenzhermeneutik als Bestimmung des spezifisch Christlichen}

Die Stellungnahmen der EKD zur Beziehung des Christentums zu anderen Religionen nehmen den Differenzgedanken explizit oder implizit auf, und zwar mit dem Ziel, das unterscheidend Christliche zu betonen. In den theologischen Leitlinien „Christlicher Glaube und nichtchristliche Religionen“21 wird „Differenz“ zunächst innertheologisch verstanden als ,andauernde Differenzierung zwischen dem, was Gott tut, und dem, was Menschen tun“, ${ }^{22}$ um diese Grundunterscheidung dann in dreifacher Hinsicht - trinitätstheologisch zu entfalten: in die Leitdifferenzen zwischen Gott und Mensch, zwischen der Gnade Gottes und dem sündigen Menschen und zwischen der vom Geist Gottes gewirkten Aneignung des Evangeliums und einer vom Menschen vollzogenen Selbstrechtfertigung im Gesetz. Diese innertheologischen Differenzbestimmungen werden dann religionstheologisch appliziert und dabei $\mathrm{zu}$ Abgrenzungsmarkern.

So konstatiert das Positionspapier hinsichtlich der zweiten Leitdifferenz, die sich auf das rettende und heilende Christusgeschehen bezieht, nicht nur einen Unterschied, sondern einen Gegensatz zu außerchristlichen Religionen, sofern diese die universale, die ganze Welt umfassende Heilswirksamkeit dieses Geschehens nicht anerkennen, sondern ihr widersprechen. ${ }^{23}$

In der EKD-Handreichung „Klarheit und gute Nachbarschaft“"24 wurde die Methode der Beziehungsbestimmung durch Differenzmarkierung auf das Verhältnis von Christentum und Islam angewendet, was zu klaren - und die Muslime teilweise verletzenden - Abgrenzungen führte.

21 Christlicher Glaube und nichtchristliche Religionen. Theologische Leitlinien, hrsg. vom Kirchenamt der EKD (Hannover: EKD, 2003).

22 Ebd., 10.

23 Zur Auseinandersetzung mit dieser Stellungnahme vgl. auch Reinhold Bernhardt, Ende des Dialogs? Die Begegnung der Religionen und ihre theologische Reflexion (Zürich: Theologischer Verlag, 2006), 280-284.

24 Klarheit und gute Nachbarschaft. Christen und Muslime in Deutschland, hrsg. vom Kirchenamt der EKD (Hannover: EKD, 2006). Zur Auseinandersetzung mit dieser Stellungnahme vgl. auch Jürgen Micksch (Hg.), Evangelisch aus fundamentalem Grund. Wie sich die EKD gegen den Islam profiliert (Frankfurt am Main: Lembeck, 2007). 


\section{Differenzhermeneutik als Wahrnehmung radikaler Alterität}

In dem von ihm herausgegebenen Buch Auf die Differenz kommt es an hat Uwe Gerber fünf Modelle interreligiöser Kommunikation im Kontext gesellschaftlicher und politischer Rahmenbedingungen einander gegenübergestellt, die auf ein Differenzanerkennungsmodell hinauslaufen. ${ }^{25}$ Gerber selbst plädiert dabei für eine „Alteritäts-Theologie der Religionen“. ${ }^{26}$

Das erste Modell etikettiert er als „Ausgrenzung des Anderen durch Monopolisierung“. Konditioniert durch die Selbstdefinition des Staates als einer kulturellen, religiösen, politischen und ethnischen Identitätsgemeinschaft wird demnach Zuwanderern zugemutet, ihre Herkunftsidentität im Sinne der Integration in diese Gemeinschaft zumindest im öffentlichen Raum zurückzunehmen. Wer sich diesem Konformitätsdruck entzieht, muss mit Ausgrenzung rechnen.

Nach dem zweiten Modell der „Duldung des Anderen bei formaler Multikulturalität“ findet eine asymmetrische, repressive Tolerierung der Minderheit durch die Mehrheit statt. Von der Minderheit wird erwartet, dass sie sich in den ihr zugewiesenen Nischen der Mehrheitsgesellschaft einrichtet, ohne dabei den Anspruch auf die aktive Mitgestaltung der Gesellschaft und politische Partizipation $\mathrm{zu}$ erheben. Im Unterschied zum ersten Modell werden die Differenzen zwischen der Mehrheitsgesellschaft und den religiösen und kulturellen Minderheiten nicht durch deren Integration aufgehoben. Sie bleiben bestehen, werden toleriert, aber nicht in eine dialogische Beziehung zueinander gesetzt. Es kommt zu keinem Austausch, sondern zur Bildung von Parallelgesellschaften. Das Interesse der Mehrheitsgesellschaft an den Minderheiten bezieht sich nicht auf deren ,inhaltliche“ kulturelle und religiöse Eigen- und Besonderheiten, sondern nur darauf, ob sie die (von der Mehrheitsgesellschaft festgelegten) formalen Regeln einer friedlichen Koexistenz einhalten. Kulturelle und religiöse Differenzen, die sich zu Spannungen aufbauen können, werden dabei nicht bearbeitet, sondern oberflächlich domestiziert. Gegenseitige Vorurteile bleiben bestehen und können sich

25 Uwe Gerber, „Interreligiöser Dialog zur Friedensförderung. Abgrenzung - Toleranz - Differenz“, in Auf die Differenz kommt es an. Interreligiöser Dialog mit Muslimen, hrsg. von Uwe Gerber (Leipzig: Evangelische Verlagsanstalt, 2006), 63-78.

26 Uwe Gerber, Wie überlebt das Christentum? Religiöse Erfahrungen und Deutungen im 21. Jahrhundert. Erlösung - Versöhnung - Erleichterung - Vereindeutigung - Alterität (Zürich: Theologischer Verlag, 2008), 191-260, bes. 256-260. Vgl. dazu Reinhold Bernhardt, „Dialog und Theologie der Religionen“, Theologische Rundschau 75 / 4 (2010): 431-435. 
wechselseitig verstärken. In den ihrer Eigendynamik überlassenen gesellschaftlichen Ghettos können sich repressive Strukturen entwickeln.

„Harmonisierung durch eine westlich-christliche Einheitskultur“ nennt Gerber das dritte Modell, das sich auf dialogische Beziehungen zwar einlässt und diese fördert, dabei jedoch einseitig am Aufsuchen von Gemeinsamkeiten interessiert ist. Der Einheitsgedanke ist leitend; er drängt das Verschiedene zurück. Die Einheit kann in Grundwerten und Menschenrechten oder in einem gemeinsamen Weltethos der Religionen gesucht werden. Dieser religiöse oder ethische Minimalkonsens soll als Bindemittel der Gesellschaft fungieren. Differenzen können dabei als störend empfunden und zurückgedrängt werden. Letztlich bestimmt die Leitkultur den Kernbestand an kulturellen, moralischen, rechtlichen und politischen Werten. Weil diesbezüglich eine grundlegende Einigkeit unterstellt wird, muss der Wertekonsens zwischen den angestammten und zugewanderten Gesellschaftsgruppen nicht mehr ausgehandelt werden. Dieses Harmonisierungsmodell kann leicht in das Ausgrenzungsmodell umschlagen, wo gesellschaftliche Gruppierungen mit dem Minimalkonsens nicht kompatibel erscheinen. Und diese Kompatibilität wird von der Mehrheitsgesellschaft festgestellt.

„Dialog“ ist der Leitbegriff des vierten Modells. Gerber sieht auch in diesem Modell die Gefahr, dass das Gemeinsame gesucht und das Differente dabei zurückgestellt wird. Im Unterschied zum dritten Modell stellt das Gemeinsame nicht den Ausgangspunkt, sondern das Ziel dar. Worin allerdings dieser Zielpunkt besteht, wird zumeist von der den Dialog initiierenden Seite vorgegeben.

Das von ihm favorisierte - offensichtlich von Emmanuel Levinas, Michel Foucault und Axel Honneth inspirierte - fünfte Modell fasst Gerber mit dem Leitwort „Dialog durch Anerkennung des Anderen in seiner Differenz“ zusammen. Es geht vom Widerfahrnis der „leibhaftigen“ Begegnung mit dem Anderen in dessen Anderssein, Eigenheit und Einzigartigkeit aus, das dem Dialog voraus geht. „Der Anstoß und die innere Verpflichtung zum Anerkennen geht immer vom Gegenüber aus, der vom anderen Menschen Anerkennung einfordert und diesen zugleich befähigt, anerkennend $\mathrm{zu}$ antworten." ${ }^{27}$ Nur so, indem der Andere in seiner Eigenständigkeit, ja Fremdheit wahrgenommen wird, kann er Gerber zufolge zum eigentlichen Subjekt des Dialogs werden. Die konkrete Differenz zwischen mir und ihm, zwischen meiner und seiner kulturellen und religiösen Prägung wird auf diese Weise zum produktiven Angelpunkt des Dialogs. Fragen wie etwa jene nach der gesellschaftlichen Integration, der politischen Partizipation oder der Ausübung von Religionsfreiheit sind Gegenstand ständigen Aushandelns. Im Vordergrund stehen dabei jeweils der individuelle Mensch

27 Gerber, „Interreligiöser Dialog zur Friedensförderung“ (wie Anm. 25), 72. 
und die soziale Beziehung, nicht jedoch die Religion oder ein Grundwertekanon. Geht es bei Duldungstoleranz um eine asymmetrische Beziehung vom eigenen zum anderen Selbst, so ist die Asymmetrie hier umgekehrt. Sie geht vom Anderen aus, der meine Wahrnehmung herausfordert. Die dabei zu Tage tretende Differenz kann auch Konflikte offenbaren oder zu solchen führen. Diese sollen nicht übergangen oder harmonisiert, sondern produktiv ausgetragen werden.

Das Differenzmodell führt Gerber zu einer Kritik an religionstheologischen Entwürfen, die auf das Gemeinsame der Religionen rekurrieren. Er nennt in diesem Zusammenhang „das harmonisierende Bild von den drei abrahamitischen Religionen“, ${ }^{28}$ also das Konzept der „abrahamischen Ökumene“, das vor allem von Karl-Josef Kuschel und Berthold Klappert propagiert und von einzelnen evangelischen Landeskirchen übernommen wurde. ${ }^{29}$ Doch seine Kritik reicht noch weiter. Sie betrifft letztlich das Dialogprogramm insgesamt. Er sieht sehr klar, dass ein konsequent zu Ende gedachter alteritätstheoretischer Ansatz die Bemühung um interreligiösen Dialog gänzlich unmöglich macht. Bestenfalls kann es demnach zu einer mehr oder weniger gelingenden Begegnung mit dem je einzelnen (religiösen) Subjekt in seiner unvergleichlichen Einzigartigkeit kommen.

Bei aller berechtigten Kritik an einer Überbetonung des Gemeinsamen als Grundlage des Dialogs kann und muss man fragen, ob das Pendel hier nicht zu weit in die entgegengesetzte Richtung ausgeschlagen ist: Nicht das Gemeinsame, sondern das Unterscheidende ist konstitutiv für die Beziehung. Letztlich begegnen sich also immer Fremde. Dieses Verständnis mag einleuchten für die Erstbegegnung von Unbekannten. Doch wenn aus dieser Erstbegegnung eine Dauerbeziehung wird, wenn Vertrautheit wächst und das Differente zu Gunsten des Gemeinsamen immer weiter zurücktritt (wenn es auch niemals gänzlich aufgehoben werden kann und darf), dann verliert ein solches Konzept an Deutungskraft.

Genau darin besteht aber das Ziel interreligiöser Dialogbemühungen: im Aufbau möglichst tragfähiger Beziehungsstrukturen und belastbarer Kommunikationskulturen. Mit der Propagierung des Modells „Dialog“ als interreligiöser Beziehungsform verbindet sich die Hoffnung, dass sich im Laufe der Zeit Wahrnehmungsformen zwischen den Beteiligten entwickeln, die dem je Anderen gerecht werden, so dass es auf dieser Grundlage zu Lebensformen einer friedlichen, aneinander interessierten und kommunikativ lebendigen Koexistenz von

28 Ebd., 75.

29 Rheinische Kirche (Landeskirchenamt, Abt. III: Ökumene), Abraham und der Glaube an den einen Gott (Düsseldorf: Evangelische Kirche im Rheinland, 2009) [http://www.ekir.de/ekir/dokumente/LS2009-B1032.pdf - Abruf: 07.05.2013]. 
Anhängern unterschiedlicher religiöser Traditionen kommen kann. Die Dynamik solcher interreligiösen Beziehungen, die über längere Zeiträume bestehen, wird in den an Levinas orientierten Alteritätsmodellen nicht ausreichend gewürdigt.

„Dialog“ ist eine spezifische Kommunikations- bzw. Beziehungsform. Sie gründet auf gegenseitigem Verstehen-Wollen und schließt dabei immer eine Form der communio, der Gemeinschaftlichkeit ein. In der Pragmatik des Dialogs werden ethische Grundhaltungen, wie gegenseitiger Respekt, die Unterstellung der Wahrhaftigkeit und die Bereitschaft zur Verständigung in Anspruch genommen. Radikal gedachte Differenz bzw. Inkommensurabilität stellt diese vorausgesetzten Gemeinschaftlichkeitsgrundlagen in Frage.

Ein wichtiges Wahrheitsmoment dieser Alteritäts-Theologie-der-Religionen besteht in der Einsicht, dass nicht Religionsgemeinschaften, Konfessionen oder gar ganze Religionen einander begegnen können, sondern immer nur Personen. Doch sind diese Personen geprägt durch ihre Religion und Konfession. Sie begegnen sich als Christ und Muslima.

\section{Differenzhermeneutik als Theorie der religiösen Selbstkonstitution durch Unterscheidung vom Anderen}

Christian Danz ist darum bemüht, religionstheologische Überlegungen aus einer philosophischen Theorie der Religion heraus $\mathrm{zu}$ entfalten. ${ }^{30}$ Er bestimmt zunächst die Problemanforderungen, die an eine gegenwärtige Theologie des religiösen und kulturellen Pluralismus zu stellen sind. Sie müsse mindestens vier verschiedene Faktoren in möglichst stimmiger Verzahnung zur Geltung bringen, und zwar die Standortgebundenheit jeder Religionstheologie, die Wahrung der Eigenständigkeit anderer Religionen, die Vergleichbarkeit der Religionen und

30 Vgl. Christian Danz, „Dogmatik als Differenzhermeneutik. Überlegungen zur Funktion moderner Systematischer Theologie im Anschluß an Ernst Troeltsch“, Kerygma und Dogma 47 (2001): 210-226; ders., ,Moderne Dogmatik und religiöse Vielfalt. Überlegungen zu einer Theologie der Religionen“, Theologische Zeitschrift 58 (2002): 140-159; ders., „Theologie der Religionen als Differenzhermeneutik. Ihre religionstheoretischen und systematischen Voraussetzungen“, in ders. und Körtner (Hg.), Theologie der Religionen (wie Anm. 17), 77-103; ders., Einführung in die Theologie der Religionen (wie Anm. 11), 221-239; ders., Die Deutung der Religion in der Kultur. Aufgaben und Probleme der Theologie im Zeitalter des religiösen Pluralismus (Neukirchen-Vluyn: Neukirchener Theologie, 2008), 127-148. 
die Gewinnung von Beurteilungsmaßstäben. ${ }^{31}$ Keiner der von ihm diskutierten religionstheologischen Entwürfe scheint ihm zufolge in der Lage zu sein, diese Anforderungen zu erfüllen.

Die Pluralistische Religionstheologie John Hicks ${ }^{32}$ unterläuft Danz zufolge die Vielfalt der Religionen, indem sie diese soteriologisch gleichnamig mache. Zumindest in den nach der „Achsenzeit“ (Karl Jaspers) - also zwischen etwa 800 und 200 v.d.Z. entstandenen Weltreligionen - lässt sich nach Hick ein strukturanaloger Grundvorgang feststellen: die Transformation des Menschen von der Selbstzentriertheit zur Ausrichtung auf die göttliche Wirklichkeit. Die Religionen können daher als verschiedene Antworten auf den einen, vom göttlichen Seinsgrund ausgehenden Transformationsimpuls verstanden werden. Damit ist nach Danz zwar eine Vergleichbarkeit zwischen den Religionen hergestellt, allerdings auf Kosten ihrer Eigenständigkeit, denn aus seiner Sicht werden sie damit zu bloßen Erscheinungsformen eines überreligiösen, gemeinsamen Kerns herabgestuft. Die Standortgebundenheit der eigenen Perspektive werde zu Gunsten einer überreligiösen Metaperspektive aufgehoben. ${ }^{33}$

Den von mir vertretenen „mutualen Inklusivismus“ kritisiert Danz, weil er es zwar ermögliche, die Standpunktgebundenheit der Religionstheologie einschließlich ihrer Bemühung um eine interreligiöse Urteilsbildung zur Geltung $\mathrm{zu}$ bringen, doch wiederum die Besonderheit anderer Religionen nicht zu würdigen vermöge. Denn wo versucht werde, aus der christlichen Binnenperspektive heraus zu einer Wertschätzung anderer Religionen zu gelangen, indem man etwa die Universalitätspotenziale der Trinitätslehre religionstheologisch fruchtbar mache, führe das unweigerlich zu einer Vereinnahmung anderer Religionen. Auch hier sei ein jenseitiger göttlicher Grund vorausgesetzt, der eine substantielle Kontinuität der Religionen verbürge. Der Unterschied zu Hick bestehe lediglich darin, dass der Erkenntnisweg zu diesem Grund nur über die je eigene Religionstradition führe. Aufgrund dieser Selbstbindung der Religionstheologie sei es weder möglich, die Religionen miteinander $\mathrm{zu}$ vergleichen, noch universalisierbare Normen für die interreligiöse Urteilsbildung zu bilden. ${ }^{34}$

Die Komparative Theologie, so Danz, werde ihrer erklärten Absicht, die Konkretheit und Andersartigkeit der Religionen zu würdigen, zwar gerecht, reflektiere aber nicht die geschichtliche Bedingtheit ihrer eigenen Position und klammere

31 Danz, Die Deutung der Religion in der Kultur (wie Anm. 30), 70, 103.

32 Vgl. dazu Reinhold Bernhardt, ,John Hick (1922-2012)“, in Arbeitsbuch Theologiegeschichte. Diskurse. Akteure. Wissensformen, Bd. 2: 16. Jahrhundert bis zur Gegenwart, hrsg. von Gregor Maria Hoff und Ulrich H. J. Körtner (Stuttgart: Kohlhammer, 2013), 356-374.

33 Danz, Die Deutung der Religion in der Kultur (wie Anm. 30), 44-53.

34 Ebd., 53-62. 
das Problem einer normativen Beurteilung von religiösen Erscheinungen aus. Die von Klaus von Stosch herausgearbeiteten Kriterien stellten eher Strukturmerkmale von religiösen Überzeugungen, nicht aber geltungstheoretische Normen dar. ${ }^{35}$

Nachdem Danz auf diese Weise die beschränkte Leistungsfähigkeit der bisher entwickelten religionstheologischen Programme aufgewiesen zu haben glaubt, setzt er zur Entfaltung seines eigenen Entwurfs an, der beansprucht, allen vier der genannten Problemanforderungen in gleicher Weise gerecht $\mathrm{zu}$ werden. Dabei geht er religionstheoretisch zu Werke, indem er zunächst über den Religionsbegriff reflektiert. Die Mängel der zuvor referierten und kritisierten Programme sind nach seiner Auffassung nicht zuletzt darin begründet, dass diesen eine tatsächliche religionsphilosophische Grundlegung fehle. Diese Grundlegung arbeitet er im Durchgang durch die neuzeitliche Problemgeschichte des Religionsbegriffs heraus, wobei ihm vor allem Kant, Schelling, Troeltsch und Tillich wichtige Gesprächspartner sind. Besonders bei Ernst Troeltsch findet er die gesuchte „Unterscheidung und Verzahnung von kulturhermeneutischer Religionstheorie mit einem normativen Religionsbegriff““. ${ }^{36}$ Anknüpfend daran will er einen Wesensbegriff der Religion und einen davon unterschiedenen Normbegriff entwickeln.

In der Durchführung dieses Programms gelangt Danz allerdings nicht über die Beschreibung von Rationalitätsstandards zur formalen Gewinnung des Religionsbegriffs hinaus. Der von ihm vorgeschlagene Wesensbegriff der Religion bleibt vollkommen abstrakt und damit für eine Religionstheologie, die von den geschichtlichen Religionen ausgeht, unbrauchbar. Danz selbst fordert diese Abstraktion, weil er sich der Einsicht nicht verschließen kann, dass jeder Wesensbegriff der Religion aus einer bestimmten religiösen Tradition heraus gewonnen ist. Um ihn als Allgemeinbegriff zur Erschließung des religiösen Feldes verwenden zu können, müsse er zumindest von seinen normativen Bestandteilen gereinigt werden. ${ }^{37}$

In dieser nicht nur von aller Normativität, sondern auch von aller Inhaltlichkeit gereinigten Form verweist der Wesensbegriff der Religion dann allerdings nur noch auf die menschliche Selbst- und Weltdeutung im Lichte des Unbedingten. Der religionsphilosophisch rekonstruierte Wesensbegriff der Religion besteht nach Danz (im Gefolge der Religionsphilosophie des Deutschen Idealismus) in der Vermittlung zwischen Bedingtem und dem Unbedingten. Erfahrung, Deutung und Unbedingtheit - diese drei Momente kennzeichnen aus seiner Sicht Religion.

35 Ebd., 62-68.

36 Ebd., 103.

37 Ebd., 106, 124. 
Von anderen kulturellen Deutungsvorgängen unterscheidet sie sich ihm zufolge darin, dass hier der kulturelle Deutungsvorgang selbst gedeutet wird. ${ }^{38}$ Religion thematisiere Differenzerfahrungen wie Krankheit, Leiden, Fremdheit, sittliche Widersprüche, ordne sie in einen umfassenderen Sinnzusammenhang ein und mache sie dadurch ertragbar. Es ist dies eine sicher zutreffende, aber rein funktionale Beschreibung von Religion, die man mit „Kontingenzbewältigung“ zusammenfassen kann. Damit bleibt Danz hinter seinem eigenen Anspruch zurück, eine substantielle mit einer funktionalen Religionsbestimmung zu verbinden.

Zur Gewinnung des Normbegriffs von Religion greift Danz auf das protestantische Religionsverständnis zurück, das durch ein Bewusstsein individueller endlicher Freiheit charakterisiert sei. Endliche Freiheit aber könne sich nur in der wechselseitigen Anerkennung der Freiheit des Anderen realisieren. Aus dem Selbstvollzug der Freiheit ergebe sich die Anerkennung von Alterität und Differenzen. „Das Andere ist Teil der eigenen Bestimmtheit. Denn nur im unterscheidenden Bezug auf Anderes kann etwas paradoxerweise es selbst sein. Bereits für die Bestimmtheit des Eigenen ist also Anderes in seiner Andersheit vorausgesetzt und in Anspruch genommen. “39 Die für die interreligiöse Begegnung grundlegende Differenzerfahrung zwischen Selbst und Anderem kann nach Danz von daher nur im Sinne einer Anerkennung des Anderen bearbeitet werden.

Diese am Selbstbewusstsein des Subjekts gewonnene Konstruktion überträgt Danz sodann auf die protestantische Religion im Allgemeinen. So wie sich das Subjekt nur im unterscheidenden Bezug auf den Anderen seiner selbst bewusst werde, so könne auch die protestantische Religion ihrer Eigenart nur in der Erfahrung der Differenz gegenüber anderen Religionen ihrer selbst ansichtig werden. Das Wechselverhältnis zwischen Selbst und Anderem kommt nach Danz somit auch bei kollektiven Geschichtsgrößen zur Geltung.

Danz' Konstruktion besteht zunächst aus einer kühnen Reduktion: Aus der Vielfalt der Bestimmungsfaktoren, die evangelischen Glauben ausmachen, schneidet er das eine Moment des Freiheitsbewusstseins heraus. Damit bindet er sich an eine subjekttheoretische Bewusstseinsphilosophie idealistischer Prägung. Indem er die Unbedingtheitsdimension auf menschliches Sinnbewusstsein beschränkt, zieht er die Differenz zwischen Mensch und Gott ein. Gott gibt es nur als menschliches Gottesbewusstsein.

Seine Konstruktion besteht sodann aus begründungslos eingeführten Postulaten. So unterstellt er erstens, dass mit dem Bewusstsein eigener, endlicher Freiheit notwendig das Bewusstsein der Anerkennung der Freiheit des Anderen

38 Ebd., 118.

39 Ebd., 125. 
verbunden sein müsse. Es ist dies eine Behauptung, die zumindest empirisch höchst bestreitbar ist. Aus dem Bewusstsein eigener endlicher Freiheit könnte sich gleichermaßen auch das Streben nach Selbstdurchsetzung gegen Andere ergeben. Zweitens geht er davon aus, dass sich die Selbstkonstitution nur durch Unterscheidung von Anderen vollzieht. Wie wir aus der Entwicklungspsychologie aber wissen, ereignet sie sich mindestens ebenso durch Identifikation mit Anderen. Drittens postuliert er, Glaube sei ein „Differenzbewusstsein, das sich in der Wahrnehmung von Differenzen realisiert“. ${ }^{40}$ Warum ist Glaube nicht auch ein Gemeinschaftsbewusstsein, das sich in der Wahrnehmung von Einheit realisiert? Ein solches Verständnis von Glauben führt $\mathrm{zu}$ dessen extremer Individualisierung, was auch innerchristlich ein erhebliches Problem darstellt, zum einen mit Blick auf die Gemeinschaftsdimension innerhalb der eigenen Glaubensgemeinschaft, zum anderen in ökumenischer Hinsicht. Viertens nimmt er als gegeben an, dass ein solches Unterscheidungsbewusstsein ein Zugehörigkeitsbewusstsein begründet. Auch hier ließe sich mit gleichem Recht das Gegenteil behaupten. Fünftens setzt er voraus, dass sich die Theorie der Selbstkonstitution durch Unterscheidung von Anderem auf ganze Religionstraditionen übertragen lässt und auch dort $\mathrm{zu}$ einem Zusammengehörigkeitsbewusstsein führt. All diese Annahmen lassen sich mit Fug und Recht bestreiten. Sie gewinnen ihre Plausibilität aus einem geschlossenen religionsphilosophischen Theoriemodell, das von empirischen Gegebenheiten absieht. Im Blick auf die real existierende Religion kommt man mit solchen geistigen Kathedralen nicht weit. Und für die Verarbeitung interreligiöser Begegnungserfahrungen tragen sie schon gar nichts aus; zumal der hier vorgelegte Entwurf (auf der Linie Ernst Troeltschs) auf die Behauptung der Höherwertigkeit des (protestantischen) Christentums gegenüber anderen Religionsformen hinausläuft. Denn in dieser Religionsform ist das von Danz geforderte aufgeklärte und reflektierte Religionsverständnis mit dem Freiheitsgedanken im Zentrum systembildend geworden, so seine Überzeugung.

Beim Ansatz von Danz handelt es sich um Religionsphilosophie, nicht aber um Religionstheologie. Die Religionen in ihrer geschichtlichen Spezifität kommen nicht in den Blick und so kann auch keine theologische Auseinandersetzung mit ihnen stattfinden. Auch die Situation des religiösen Pluralismus begegnet in diesem Ansatz nur als abstrakte Vorstellung, fern ab von religionsphänomenologischen und -soziologischen Befunden. Um eine Theorie an der Wirklichkeit zu bewahrheiten (oder zu falsifizieren), bedarf es eines - möglichst unverstellt wahrgenommenen - Bewährungsfelds. Bei Danz’ Religionstheorie lässt sich ein

40 Ebd. 
solcher Bezug nicht erkennen. Sie kann bestenfalls auf ihre innere Kohärenz befragt werden. Und selbst diese erscheint nach den oben vorgetragenen, kritischen Überlegungen fragwürdig. Man müsste diese Theorie vom Kopf auf die Füße stellen, doch was bliebe dann noch von ihr übrig?

\section{Differenzhermeneutik als Hermeneutik der Differenz}

Der letzte in diesem Artikel zu besprechende Ansatz der Differenzhermeneutik ist von den postmodernen Differenzphilosophien (Lyotard, Derrida u.a.), von Foucaults Analysen hegemonialer Diskurse sowie von semiotischen Konzepten beeinflusst. Er wird von Gregor Maria Hoff vertreten. ${ }^{41}$ Hoff entwickelt den differenzhermeneutischen Ansatz fundamentaltheologisch, materialdogmatisch, ökumenisch, israeltheologisch und religionstheologisch.

Fundamentaltheologisch beschreibt er die Grundsituation des Verstehens semiologisch als fortwährendes Verweisen von Zeichen auf Zeichen. Damit aber werden immer neue Differenzen gesetzt, die neue Verstehensvorgänge erfordern. Das Verstehen erzeugt also das zu Verstehende. Die Differenz, der Zwischenraum zwischen den Zeichen, ist der Ort des produktiven Verstehens. Die in theologischen Redeformen verwendeten Zeichen verweisen zudem auf die Dimension des Unsagbaren, das ihnen voraus liegt und das von den Zeichen niemals eingeholt werden kann. Diese Differenzrelation ist konstitutiv für Theologie und führt mit innerer Notwendigkeit zu einer Vielfalt des Sprechens von Gott. Theologie kann es nur im Plural geben. ${ }^{42}$ Und „Spurenelemente christlicher Theologie“ lassen sich auch jenseits des kirchlich normierten Sprachstroms finden..$^{43}$

Auch materialdogmatisch setzt Hoff bei Differenzialen an, die zu unterschiedlichen Interpretationen Anlass geben und damit Generatoren theologischer Produktivität und Kreativität waren und sind. So fungierte und fungiert die Eschatologie etwa als theologisches Korrektiv gegenüber Gottesbildern sowie

41 Gregor Maria Hoff, Die prekäre Identität des Christlichen. Die Herausforderung postmodernen Differenzdenkens für eine theologische Hermeneutik (Paderborn [u.a.]: Schöningh, 2001); ders., Ökumenische Passagen - zwischen Identität und Differenz. Zum aktuellen Stand des ökumenischen Gesprächs zwischen römisch-katholischer und evangelischer Kirche (Innsbruck: Tyrolia, 2005); ders., Stichproben: Theologische Inversionen. Salzburger Aufsätze (Innsbruck: Tyrolia, 2010), darin besonders die Artikel 3, 4, 12, 17.

42 Hoff, Stichproben (wie Anm. 41), 225-233.

43 Ebd., 231. 
Sprach- und Praxisformen des Gottesglaubens. Die Differenz zwischen universalund ortskirchlichen Traditionen stimulierte und stimuliert die Ekklesiologie. Die Logik und Pragmatik der (in der Dogmatischen Konstitution „Lumen gentium“ des Zweiten Vatikanischen Konzils getroffenen) Feststellung, dass die geistliche Kirche Jesu Christi in der institutionell verfassten römisch-katholischen Kirche „verwirklicht“ sei (subsistit), provozierte und provoziert etwa unterschiedliche Positionsbestimmungen in der ökumenischen Theologie.

Als ökumenisch entscheidend bezeichnet Hoff die Frage, ob sich der eigene konfessionelle Wahrheitsanspruch als so differenzfähig erweist, dass er eine Vielfalt von Wahrheitserschließungen als notwendige Folge der Unverfügbarkeit der Wahrheit ansieht, auf die sich die Theologie ausrichtet. Ohne deren Wahrheitswert zu bestreiten, müssten diese - wie Fremdsprachen - in den Bezugsrahmen der eigenen Theologie übersetzt werden, so die Forderung, die sich für ihn daraus ergibt. Auch hier ist es wieder die Differenz, die über alle positionellen Interpretationen hinaus auf die letztlich uneinholbare Wahrheit verweist. ${ }^{44}$

In der Israeltheologie wird eine Differenz bearbeitet, die in einer gemeinsamen Heilsgeschichte spielt. Hoff geht davon aus, dass die Gottessprache Israels offenbarungstheologische Qualität besitzt, weil sie in einer Selbstmitteilung Gottes gründet. Es gibt demnach jüdisch wie christlich eine Teilhabe an der Fülle der Offenbarung. Weil aber Offenbarung immer Heilsoffenbarung ist, muss gelten: „Es gibt ein Heil für Israel ohne Christus-Bekenntnis.“ “45 Die Erkenntnis, dass es authentische Gottesoffenbarung extra Christum (wenn auch nicht unabhängig von Christus, sondern auf ihn hingeordnet) gibt, ist nun aber für die Theologie der Religionen insgesamt von Bedeutung. Auf diese Weise muss die Israeltheologie als Einfallstor jeder Religionstheologie begriffen werden. ${ }^{46}$

Die Religionstheologie muss Hoff zufolge davon ausgehen, dass sich Gott in einer differenten Vielfalt von Gottesauffassungen offenbart. Sie muss ein Bewusstsein für die anonyme, unerkannte, fremde Gegenwart Gottes schaffen. Dies kann pneumatologisch gedacht werden: Der Geist Gottes öffnet den Raum für unterschiedliche Formen der Gotteserkenntnis. Unter Bezugnahme auf $\S 7$ des Dekrets über die Missionstätigkeit der Kirche „Ad gentes“ formuliert Hoff als Grundüberzeugung seines religionstheologischen Ansatzes: „Gott schafft Heil auf Wegen, die nur er kennt.“ Auch hier - in der Differenzrelation zu anderen Religionen - wird die Differenz zum privilegierten Ort der Selbst- und Gotteserkenntnis. Es kann an diesem Ort zu unerwarteten Erschließungen Gottes kommen. „Die

44 Ebd., 182.

45 Ebd., 66.

46 Ebd., 65. 
Anonymität Gottes ist der unerwartete Modus seiner heilvollen Gegenwart. Dies ist eine unerwartete Einsicht mit eigenem Offenbarungscharakter.“47

In den Differenzen der christlichen Konfessionen und außerchristlichen Religionen müsse, so lässt sich Hoffs hermeneutische Grundforderung zusammenfassen, die Bedeutung des (katholischen) Glaubens bestimmt werden. Seine Differenzhermeneutik zielt also nicht nur auf die Würdigung des Andersseins des/ der Anderen und nicht nur auf ein profiliertes Selbstverständnis, sondern auf ein dabei immer mitzuführendes Bewusstsein der unauflösbaren Geheimnishaftigkeit dessen, worum die Theologie in immer neuen Verstehensbemühungen kreist.

\section{Rückschau}

Da eine Zusammenschau der vorgestellten differenzhermeneutischen Ansätze kaum möglich ist, weil die Unterschiede und Gegensätze zwischen ihnen so groß sind, beschränke ich mich auf eine knappe, diese Differenzen, aber auch Gemeinsamkeiten benennende Rückschau.

In einigen der Entwürfe (besonders bei Hoff, Danz und Gerber) sind die Verwendungsweisen des Begriffs „Differenzhermeneutik“ von philosophischen Konzepten (vor allem von Levinas, Lyotard, Derrida, Foucault) inspiriert, in manchen so stark, dass sie nur von diesen Ansätzen her verständlich werden und deren Anwendung auf die Deutung der Religionsvielfalt darstellen. Bei Sundermeier steht die hermeneutische Tradition der westlichen Kultur - besonders Hans-Georg Gadamer - im Hintergrund. In den kirchlichen Stellungnahmen dagegen wird der Differenzbegriff ohne philosophischen Bezug und auch ohne inhaltliche Näherbestimmung gebraucht.

Die vorgestellten Entwürfe folgen unterschiedlichen Interessen: Sundermeier ist an der hermeneutischen Frage interessiert, wie Verstehen des Fremden möglich ist. Seine Xenologie zielt auf interreligiöse Verständigung, also letztlich auf eine Aufhebung der Differenzen in gemeinschaftlichen Lebensformen. Körtner will die bestehenden Differenzen nicht aufheben oder verdecken, dekonstruiert sie aber, indem er sie an Standort und Perspektive des jeweiligen Betrachters zurückbindet. Er wehrt sich gegen religionsübergreifende Grundlegungen der Religionstheologie und setzt stattdessen binnenchristlich an. Die EKD-Stellungnahmen wollen das Spezifische des christlichen Glaubens durch Differenzbestimmungen zu anderen Religionen im Allgemeinen und zum Islam

47 Ebd., 64. 
im Besonderen herausarbeiten. Demgegenüber hatte man von kirchlicher Seite in der Beziehung zum Judentum stets eher das Gemeinsame und Verbindende betont. Gerber betont die radikale Alterität des begegnenden Individuums, wobei ihm der interreligiöse Dialog insgesamt fragwürdig wird. Danz hat eine eher religionsphilosophische Agenda. Sein Ansatz zielt auf eine religionstheoretische Grundlegung der Religionstheologie, im Grunde der Theologie insgesamt. Er will den aus einem bestimmten Strang der protestantischen Tradition abstrahierten Religionsbegriff normativ fruchtbar machen. Religionsformen sind danach $\mathrm{zu}$ beurteilen, ob und wie sie das Bewusstsein individueller endlicher Freiheit zur Geltung bringen. Hoff schließlich dekliniert das Differenzparadigma durch die gesamte Systematische Theologie - einschließlich der ökumenischen Theologie, der Israeltheologie und der Religionstheologie.

Die meisten der vorgestellten Entwürfe zielen auf eine Würdigung des Andersseins des (religiös) Anderen ab, einzelne sind demgegenüber bestrebt, das Proprium der eigenen Religion herauszustellen. Allen gemeinsam ist eine skeptische Grundhaltung gegenüber einer zu starken Betonung des Gemeinsamen und die Religionen Verbindenden, wie sie vor allem in der Position des religionstheologischen Pluralismus ausgemacht wird. Demgegenüber soll die Dialektik von Identitäts- und Alteritätsbewusstsein herausgearbeitet werden. Nicht nur in den kirchlichen Stellungnahmen, sondern auch bei Danz führt das in die Nähe einer Apologetik des christlichen Glaubens bzw. der protestantischen Religion.

Die dargestellten Entwürfe streben nicht einen interreligiösen Vergleich von einer „neutralen“ Beobachterposition aus an. Sie bekennen sich zu ihrer Standortgebundenheit in einer bestimmten religiösen Tradition, setzen die Differenzrelation dementsprechend zwischen dem „Eigenen“ und dem „Anderen“ bzw. „Fremden“ an und verstehen diese Relationen als Ort theologischer Selbstvergewisserung. „Differenz“ bezieht sich auf das Anderssein der anderen Religionsform gegenüber der eigenen und „Differenzhermeneutik“ auf die Bearbeitung dieser Alteritätserfahrung. Die Beschreibung und Reflexion erfolgt also - mehr oder weniger betont - aus der Teilnehmendenperspektive.

Wie alle Beiträge zur Religionstheologie, so bearbeitet auch die Differenzhermeneutik die Spannung zwischen Partikularität und Universalität bzw. zwischen Konfessionalität und religiöser Pluralität. Sie zielt dabei weniger auf die Ebene dialogpraktischer Überlegungen und auch nicht auf die Behandlung materialdogmatischer (etwa christologischer) Fragen, die sich in der Begegnung mit anderen Religionen stellen, als vielmehr auf die Ebene des Verstehens. „Differenzhermeneutik“ zeigt eine dreifache Verstehensaufgabe an, wobei die drei Pole unterschiedlich akzentuiert sein können: Es geht ihr um das Verstehen des Anderen, das Selbstverstehen und das Verstehen der Differenz zwischen dem Selbst und dem Anderen. 